\title{
Repurposing asynchronous video interview software for virtual pharmacy OSCEs during the COVID-19 pandemic
}

\author{
Denise L. Hope (iD 1, Sean R. Alcorn (ID) 1, Gary D. Rogers (D) 2, Gary D. Grant (iD 1, Michelle A. King (D) 1 \\ 1 School of Pharmacy and Medical Sciences, Griffith University, Gold Coast QLD, Australia \\ ${ }^{2}$ School of Medicine, Deakin University, Geelong VIC, Australia
}

Keywords
Clinical competence
Education
Pharmacy
Self-correction
Self-reflection
Video recording
Correspondence
Denise L. Hope
School of Pharmacy and Medical Sciences
Clinical Sciences 2
Gold Campus
Griffith University
QLD 4222
Australia
d.hope@griffith.edu.au

Keywords

Clinical competence

Self-correction

Self-reflection

Correspondence

Denise L. Hope

Clinical Sciences 2

Gold Campus

QLD 4222

d.hope@griffith.edu.au

\begin{abstract}
Background: The COVID-19 pandemic required pharmacy educators to seek creative and innovative ways of delivering learning outcomes in the virtual environment. Objective Structured Clinical Examinations (OSCEs) have been particularly challenging to deliver online. Objective: To explore the use of asynchronous video interview software as a platform for virtual OSCEs in pharmacy education, and for conducting brief structured research interviews. Method: Fourth year Bachelor of Pharmacy students at an Australian university, were interviewed via asynchronous video interview software (Big Interview) about their perceptions of using the platform for virtual OSCEs. Results: 37 students (92.5\%) used Big Interview to complete at least one summative OSCE case during a team-based simulation. Seven research interviews were recorded by six students. Positive comments focussed on facilitation of self-reflection and selfcorrection in patient questioning and counselling. Negative views concerned the lack of authenticity, including the robotic and artificial nature of interactions. Conclusion: Asynchronous video interview software for pharmacy OSCEs may assist formative learning and self-reflection, offering an adjunct to, but not replacement of, in-person communication.
\end{abstract}

\section{Introduction}

The sudden impact of the COVID-19 pandemic on academia has been profound. Many academics were required to convert traditional face-to-face teaching to the virtual realm, almost instantaneously. These changes required the use of digital software and online platforms, several of which were commonly used in teaching, while others were designed or adapted to fill the gaps in functionality that suddenly became imperative. Yet the rapid change to virtual teaching was characterised by uncertainty for many, regarding availability of funding, academic staff, and other vital resources (Higbea et al., 2020).
In health professional education, learning and assessment activities such as objective structured clinical examinations (OSCEs), used to evaluate communication and other skills, are traditionally delivered and assessed in person, often utilising simulated patients (SPs) (Bowers et al., 2017). In pharmacy education, OSCEs often simulate pharmacist interactions with patients or other members of the health team (Austin et al., 2003). The use of OSCEs in teaching and assessment contributes to Australian pharmacy programme accreditation requirements through provision of opportunities to develop skills and confidence in interpersonal and interprofessional communication (Australian Pharmacy Council Ltd., 2020). The rapid 
change to virtual delivery prompted innovation and creativity among educators to continue to deliver the learning outcomes associated with OSCEs (Higbea et al., 2020).

Previous research reviewed the use of asynchronous versus synchronous methodologies in pharmacy education, and ultimately recommended a blended approach, offering both asynchronous and synchronous learning activities (Motycka, St Onge, \& Williams, 2013). Synchronous learning involves simultaneous interaction between two or more participants and may utilise online chat or videoconferencing. In contrast, asynchronous learning allows communication to occur over a period of time but may be less personal than synchronous learning and requires self-discipline. It may utilise streaming video and web-based activities (Motycka et al., 2013).

Online or virtual delivery of pharmacy and other health professional education, particularly in response to the pandemic, has involved the use of numerous meeting and telecommunication platforms. Synchronous OSCEs have reportedly been conducted in Google Meet and Google Classroom (Rahman, Nazar, \& Elnaem, 2020), Zoom (Kakadia, Chen, \& Ohyama, 2020; Lara et al., 2020; Major et al., 2020), Cisco Webex and Microsoft Teams (Higbea et al., 2020). One simulation-based option utilised by some universities to deliver virtual OSCEs was Education Management Solutions (2020), which hosts a pharmacyspecific virtual OSCE and a pharmacy-specific simulation. While there is currently a dearth of literature on asynchronous OSCEs, Ali (2020) reported the use of the simulation learning system, MyDispense, to deliver asynchronous web-based pharmacy OSCEs during the COVID-19 pandemic. The author reported student satisfaction with the 'tailored detailed instant feedback' of the web-based OSCE and student dissatisfaction with the perceived lack of face-to-face interaction (Ali, 2020).

Employee recruitment and selection is an area that has increased its use of asynchronous online video interview platforms since the COVID-19 pandemic. The job screening method increases the efficiency of the hiring process. It usually involves potential candidates recording their answers to predetermined questions, without the presence of a human interviewer (Rubinstein, 2020). Examples of these video interview platforms include HireVue, Modern Hire and Big Interview.

Academic staff at Griffith University had access to Big Interview (Skillful Communications Inc., 2020) and explored the opportunity to utilise it for asynchronous recording of pharmacy student assessments during the COVID-19 pandemic. Its initial application involved students completing a viva voce assessment in Big Interview in a Master of Pharmacy pharmacotherapeutics course, uploading video answers to the pre-recorded clinical questions posed by the lecturer. Unlike the later OSCEs, these cases were not simulated patient cases. To preserve academic integrity, all students undertook the pharmacotherapeutics assessment simultaneously while the lecturer was able to mark each student asynchronously, and judge communication skills, in addition to clinical knowledge. Subsequently, several other courses conducted assessments in Big Interview that involved students' video responses to posed case scenarios. Perceived potential advantages of this approach were to reduce academic staff time and repetition, standardise question delivery and reduce potential assessor bias.

The successful application of Big Interview for assessments prompted the academic team to consider trialling the platform for asynchronous OSCEs, to provide a contingency during this challenging year, in case the availability of funding, staffing and SPs became limited. While it was recognised that an asynchronous platform would lose the authenticity of a face-to-face OSCE (Ali, 2020), it was deemed to be a logical and sustainable OSCE alternative to enable students to practise and review their patient-directed verbal communication skills during the extraordinary and sudden move to online teaching as a consequence of the COVID-19 pandemic.

This paper presents the atypical use or repurposing of an asynchronous video interview software platform, Big Interview (Skillful Communications Inc., 2020), to deliver virtual formative and summative OSCEs in an Australian pharmacy programme.

\section{Objective}

To explore the atypical use of asynchronous video interview software as a platform for virtual OSCEs in pharmacy education, during the COVID-19 pandemic. Also, to trial the use of asynchronous video interview software as a platform for conducting structured asynchronous research interviews.

\section{Methods}

The exploratory study involved structured asynchronous interviews with fourth year Bachelor of Pharmacy students at an Australian university. Institutional ethical clearance was received (HREC No. 2016/594). 


\section{Pre-recorded simulated patient videos uploaded in sequence to Big Interview}

\begin{tabular}{|l|l|l|l|l|}
\hline $\begin{array}{l}\text { Patient makes } \\
\text { request }\end{array}$ & $\begin{array}{l}\text { Patient reply re: } \\
\text { nature of request }\end{array}$ & $\begin{array}{l}\text { Patient reply re: } \\
\text { med history }\end{array}$ \\
\hline $\begin{array}{l}\text {-Student asks } \\
\text { about nature of } \\
\text { request }\end{array}$ & $\begin{array}{l}\text {-Student asks } \\
\text { about med } \\
\text { history }\end{array}$ & $\begin{array}{l}\text {-Student asks if } \\
\text { patient has } \\
\text { questions }\end{array}$ \\
\hline
\end{tabular}

Student questions and counselling recorded directly into Big Interview

Figure 1: Flow diagram of typical Big Interview OSCE process

\section{Pre-recorded simulated patient and prescriber videos uploaded in sequence to Big Interview}

\begin{tabular}{|l|l|l|l|l|}
\hline $\begin{array}{l}\text { Patient makes } \\
\text { request }\end{array}$ & $\begin{array}{l}\text { Patient reply re: } \\
\text { nature of request }\end{array}$ & $\begin{array}{l}\text { Patient reply re: } \\
\text { med history }\end{array}$ & $\begin{array}{l}\text { Patient reply re: } \\
\text { questions }\end{array}$ \\
\hline $\begin{array}{l}\text { - Student asks } \\
\text { about nature of } \\
\text { request }\end{array}$ & $\begin{array}{l}\text { - Student asks } \\
\text { about med } \\
\text { history }\end{array}$ & $\begin{array}{l}\text { - Student asks if } \\
\text { patient has } \\
\text { questions }\end{array}$ & $\begin{array}{l}\text { Student contacts } \\
\text { resolution of MRP }\end{array}$ \\
\hline
\end{tabular}

Student questions, prescriber contact and counselling recorded directly into Big Interview

Figure 2: Flow diagram of Big Interview OSCE process with prescriber interaction

\section{Intervention}

In a virtually delivered final capstone semester of an undergraduate pharmacy programme, an asynchronous video interview platform (Big Interview) was used to deliver formative OSCEs for pharmacy practice workshops and summative OSCE cases in a team-based simulation. In total, 16 different formative workshop OSCE cases and 23 summative simulation OSCE cases were modified and prerecorded for delivery in Big Interview. The modification of each OSCE case involved editing the patient scenario to provide the answers to potential student pharmacist questions in sections, which were then pre-recorded in separate videos by academic or professional university staff, acting as SPs. A typical scenario progressed in a predetermined sequence (Figure 1).

If a case required identification of a medication related problem (MRP), it was configured to allow for an additional video recording, in which the student could present the MRP to the simulated prescriber and offer a resolution (Figure 2).

The patient videos were accessed in Big Interview upon provision of an assignment code. Students could incrementally view the patient videos and respond by recording their information gathering questions and medicine counselling for the patient.

Formative virtual pharmacy practice workshops were conducted in Blackboard Learn 9.1.3 Collaborate Ultra. The OSCE workshops involved four Big Interview cases, per week, for four weeks, to replace previous face-to-face OSCE practice that had utilised professional SPs. The settings in Big Interview were applied to allow multiple student attempts, enabling review and up to three attempts to record the videos. During the online workshops there was some reticence by students to partake in the virtual OSCEs and subsequent engagement was low. The course facilitators modified the intended workshop approach and shared the patient videos of one or two Big Interview cases per week to the whole class, encouraging sequential and anonymous student questions and discussions to occur in the virtual classroom whiteboard space.

An extended three-week gamified simulation was a key feature of the final semester of a four-year pharmacy programme. The team-based simulation, usually conducted in person, was converted to the virtual learning space in 2020, as a consequence of the COVID-19 pandemic. As there was uncertainty as to the availability and utility of SPs during this time, it was planned to 
convert as many of the usual OSCE cases $(n=32)$ to Big Interview, to have available as backup, should SPs or staff not be available. Ultimately, 23 OSCE cases were identified as being candidates for conversion to the Big Interview process and were modified and built in the platform. Suitable candidate cases were those that could be broken into sections for the sequential recording and delivery of patient responses. Cases that were deemed unsuitable for Big Interview were those that required other elements of face-to-face simulation, such as device demonstration, or blood pressure measurement. During the live online teambased simulation in September 2020, one or two of the asynchronous Big Interview OSCE cases were made available each day, to complement the live synchronous OSCE cases that were conducted in Microsoft Teams. The cases were conducted by an individual student, on behalf of their team. In total, 17 of the pre-recorded Big Interview cases were deployed in the simulation. These summative cases were designed to allow each team single attempts of video recording, placing more pressure on respondents to deliver their questions and counselling in one go. In contrast to the team-based summative simulation OSCEs, individual student summative OSCES were later conducted online using a more traditional synchronous approach, in Microsoft Teams, requiring more academic resources, including pharmacist assessors and SPs.

\section{Sampling and Recruitment}

Fourth year pharmacy students who had viewed or attempted at least one Big Interview OSCE case were invited to complete brief asynchronous video interviews in Big Interview, upon completion of the formative workshop OSCE cases and again upon completion of the summative simulation cases. Students were given a $\$ 10$ e-gift card as thanks for their participation in each stage of the interviews.

\section{Interview}

The Big Interview platform was also employed to deliver the brief structured interviews, as a potentially novel approach to conducting asynchronous research interviews. It was intended that the Big Interview platform might provide a familiar, accessible and non-threatening avenue for students to complete structured interviews. It was an asynchronous, no touch option, which presented a novel method for collecting answers to open-ended questions that provided a COVID-safe alternative during the pandemic. Further perceived advantages were reduction in researcher time, guaranteed interview consistency and lack of interviewer influence or bias. It was anticipated that a verbal research interview may provide less stringent participant responses than a questionnaire, avoiding the constraints of spelling and grammar.

Each interview consisted of five questions, which were recorded to video by a non-academic staff member. Questions explored the student's experience of using Big Interview for virtual OSCEs, the positive and negative aspects of the approach, opinions on being able to record multiple times or once only, and overall experience in comparison to face-to-face OSCEs.

The video interviews were transcribed verbatim by the chief investigator and quality checked by a member of the research team. Transcriptions were read and reread by two members of the research team, to generate codes and apply them, as per conventional content analysis (Hsieh \& Shannon, 2005). Concepts and themes that emerged included positive and negative aspects of using Big Interview for virtual OSCEs, and themes around authenticity of learning. All team members reviewed the analysis.

Student access to, and engagement with, the Big Interview cases was recorded and ranked from 1 to 6 , based on degree of exposure, with 1 being no exposure and 6 being exposure to case completion in workshops and the simulation. Exposure was correlated with individual student summative OSCE mark (Spearman's Correlation, SPSS 25, significance level $p<0.05$ ).

\section{Results}

\section{Participation}

Across four weeks of formative OSCE workshops, $75.0 \%$ of students $(n=30)$ accessed Big Interview. Seven students $(17.5 \%)$ completed at least one full case in Big Interview (i.e. recording at least four questioning and counselling videos). Nine students (22.5\%) completed at least one partial case (i.e. recording at least one of the videos for at least one case); 14 (35.0\%) had viewed at least one case in Big Interview but recorded no videos. Ten students (25.0\%) did not individually access Big Interview for workshop cases at all. In the summative simulation, 92.5\% of students ( $n=37$ ) accessed Big Interview, completing at least one full case each. While the lowest individual mean summative OSCE results (74.3\%) were from the group that did not access Big Interview during workshops but 
completed a case in the simulation, no statistical correlation was found between individual OSCE results and degree of exposure $\left(r_{s}=0.42, p=0.798\right)$.

\section{Interviews}

37 of 40 final year pharmacy students had viewed at least one Big Interview OSCE case and were invited to participate in the brief research interviews. Seven interviews were completed by six students (four females, two males), providing a $16.2 \%$ response rate. The gender mix of respondents (66.7\% female) aligned with that of the cohort ( $n=28,70.0 \%$ female). Five interviews were completed following virtual workshops, in August and September 2020, with mean interview duration of 2:01 minutes (minimum 1:17, maximum 3:03 minutes). The interviewees had varying exposure to Big Interview during workshops, with one student completing one or more full case/s (rank 6), two partially completing one or more case/s (rank 5), one having low exposure (rank 4) and another having no individual access during workshops (rank 3). Two further interviews were completed followed the virtual simulation, in October 2020, with mean interview duration of 3:24 minutes (minimum 2:21, maximum 4:27 minutes). Following the simulation, the interviewees had varying exposure to Big Interview, with one having completed cases in both workshops and the simulation (rank 6) and the second having only partial workshop exposure plus case completion during the simulation (rank 5).

Analysis of interviews revealed several key concepts and themes related to the positive and negative aspects of using Big Interview for virtual OSCEs. Positive aspects included the ability to review the videos to self-reflect and self-correct patient questioning and counselling, the flexibility of the platform and the capacity to plan communication. Negative aspects reported focused on technical or procedural challenges, and the lack of authenticity, including the robotic or artificial nature of interactions. Exemplar interview comments are summarised against these themes in Table I.

In addition to the main themes identified, several students also discussed the impact of the virtual OSCEs on their confidence:

'Talking to the screen when there's not actually someone there has been really good to help with things I'm less sure about.' [P3]

I thought that was really good to help build my confidence.' [P2b]
Table 1: Main positive and negative aspects of Big Interview OSCES

\begin{tabular}{|c|c|c|}
\hline & Theme & Exemplar Interview Quotation \\
\hline $\begin{array}{l}\text { Positive } \\
\text { Aspects }\end{array}$ & Self-Reflection & $\begin{array}{l}\text { 'And it helps you learn; you get to see what } \\
\text { the answers that you missed were.' [P1] } \\
\text { 'I think it's helped me develop my own self- } \\
\text { reflective skills as well, so I got to have a } \\
\text { look and listen to how I say things and I } \\
\text { could instantly put that feedback into } \\
\text { practice and try and give it another } \\
\text { go.' [P3] } \\
\text { 'I liked it because I could replay and listen } \\
\text { to what I was saying and then I could } \\
\text { update my responses to make them sound } \\
\text { more professional or more comprehensive.' } \\
\text { [P5] }\end{array}$ \\
\hline
\end{tabular}

Self-Correction 'I could keep retrying and deleting and rerecording the videos when I needed to, to perfect my work.' [P2a]

'I found it beneficial because if I had forgotten a question that I should've asked then I could redo it and review.' [P4]

Flexibility I've been using it to practise my skills... in the privacy of my own home and on my own, as independent learning.' [P2a]

'It's been a really great way to still practise verbalising counselling and question taking whilst at home in the virtual environment.' [P3]

'In the new online learning environment, it recreated an element of patient interaction that would otherwise be difficult to simulate.' [P5]

P I a n n i n g 'It was a lot less stressful... because I was Communication able to prepare appropriately, and it didn't feel like someone was lingering and watching me while I prepared my notes.' [P2b]

'It also helped me create a sense of logic as to what sort of questions / should be asking and how to go about those questions.' [P4] 'It was good to be able to... listen to it over again, and plan. And, so therefore, it's a very good way of improving what you say during a clinical interaction.' [P5]

Negative Technical or 't takes a long time to upload, that's a Aspects Procedural negative.' [P1]

Challenges 'I experienced a few glitches with Big Interview where it wouldn't upload the video after I made it, so... that was a bit stressful at the time.' [P2b]

'The major downside of the Big Interview is, firstly, getting used to how it works.' [P5]

L a ck of 'It still feels a bit robotic, press the play Authenticity button, it doesn't feel like an actual real-life interaction.' [P1]

'It's not like a flowing conversation. So, it doesn't feel like I'm actually talking to anybody, but it's more like I'm practising for a speech.' [P2a]

'Big Interview did feel a bit unnatural at times because we weren't getting a response from the patient live, so we couldn't really follow the narrative of how the OSCE was going.' [P3] 
Another student commented on the authenticity of the summative approach to the virtual OSCEs in Big Interview, when only a single recording attempt was allowed:

'In practice you've only got one chance to say the right thing to the patient. You don't have a redo... and in that manner it closer reflects practice.' [P6]

\section{Discussion}

This study identified that final year pharmacy students who accessed virtual asynchronous OSCEs in Big Interview valued the ability to self-reflect and self-correct their verbal patient questioning and counselling skills. The study revealed positive and negative aspects of the approach, and clear shortcomings in use of the platform for conducting research interviews.

\section{Use of platform for OSCES}

Student engagement with Big Interview virtual OSCEs was low, particularly with the formative workshop OSCE cases. This may be attributed to a lack of acceptance of the interface, the fact that some cases were presented to the whole cohort in workshop teaching or possibly to perceived student fatigue with virtual learning toward the end of 2020. However, there was no avenue in the research to discern why the non-users did not engage with the platform.

Students who accessed the asynchronous virtual OSCES expressed positivity about the flexibility the platform provided, to enable independent access to view patient videos, and respond if they chose, in the privacy and security of their home environments. The flexibility of asynchronous case access therefore appears to confer a degree of autonomy on students, to control the time and location of their learning. Autonomy is a positive attribute of experiential education (Higbea et al., 2020) and is argued to be something craved by students and met by blended learning approaches, involving asynchronous and synchronous activities (Motycka et al., 2013). Supporting student autonomy may help to improve student motivation and the development of professional identity (Mylrea, Sen Gupta, \& Glass, 2017).

Another identified strength was the ability for students to plan out their communication, in advance of recording their responses. This attribute strongly aligns with traditional uses of the asynchronous video interview platforms for employment interviews, in which par- ticipants can apply intellectual skills such as problem assessment, judgement, decision-making, critical thinking and planning (Gorman, Robinson, \& Gamble, 2018). Several students also reported that using the virtual OSCE cases helped to improve their confidence. This well aligns with the intended learning outcomes for OSCEs and the team-based simulation. OSCEs have a long history of being used in health professional education to improve student confidence (Ytterberg et al., 1998).

Strongly positive themes in this study were students' perceived ability to self-reflect and self-correct their communication techniques. They reported the benefit of being able to play their videos back, to review and reflect upon their verbal communication. This aligns with previous research conducted in physiotherapy education, where student reflection on supplemental self-video was associated with improved OSCE performance (Maloney et al., 2013). There were several positive participant comments about the opportunity to rerecord and perfect their work. This approach of self-correction strongly aligns with the design principles of the Montessori educational method (Marshall, 2017) and is congruent with overarching self-determination theory (Mylrea et al., 2017). When replaying their work and critically reviewing it for potential improvement, the student is arguably placing themselves in the simulated role of the pharmacist, which supports development of professional identity (Mylrea et al., 2017). Authenticity of the task is also important to the development of professional identity, however, the lack of real-world conversation in the virtual OSCEs generated a recognised lack of authenticity and may have contributed to the poor student engagement with the platform.

A primary negative theme to emerge from the participant interviews was the lack of authenticity, or real-life communication exchange, even though this was an acknowledged limitation of the approach out the outset of the course. Students described the lack of conversational exchange as one-sided, robotic and unnatural. While authenticity of the task is strongly related to student motivation and professional identity (Mylrea et al., 2017), the use of the asynchronous virtual OSCEs in Big Interview or other platforms may serve as a valuable adjunct to traditional OSCE teaching methods, allowing for flexible delivery and independent access to learning resources, as was required during the COVID-19 pandemic.

The other negative aspects identified in interviews were occasional technical and procedural challenges. Students' experience of technical difficulties with online technologies has been commonly reported prior to and during the COVID-19 pandemic (Archibald et al., 2019; 
Kakadia et al., 2020). The sudden shift to online delivery of education forced students and educators alike to embrace digital technologies, irrespective of their degree of digital literacy and comfort. While one participant suggested that it took time to become familiar with the process, most other students reported that the virtual OSCEs were easy to navigate.

This study has suggested that virtual OSCEs conducted in an asynchronous video interview platform might provide a sustainable and valuable adjunct to traditional OSCE methods. The sustainable benefits derive from the fact that once the cases are video recorded and loaded sequentially into the interview platform, they can be accessed repeatedly by subsequent student cohorts, reducing the need for future staff or SPs for delivery. Potential student value derives from their ability to access the cases independently, providing autonomous control over their own learning. The approach also enabled critical self-reflection and self-correction, which may improve confidence and competence in verbal counselling, and offer potential avenues for remediation of learning. The approach may well suit lower year levels of pharmacy students, where arguably the fidelity of simulation is less important than scaffolding students' confidence and selfreflection to develop OSCE skills. Using an asynchronous video interview platform may provide less confrontational ways to practise history taking, patient questioning and counselling, allowing students to plan and reflect on their communication. Furthermore, the approach may provide an important avenue for remediation of struggling students as co-curricular or extra-curricular support.

\section{Use of platform for research interviews}

The novel research approach of using the Big Interview platform to deliver the asynchronous research interviews was convenient and easy to use. This approach is used ubiquitously in employment interviews and previous research attempted to validate their use in the employment sector (Gorman et al., 2018). However, limitations identified in the Gorman et al. study included potential for bias due to their use of self-reports of job performance rather than an independent rating.

Response rate to the research interviews was low, but closely aligned with the response rate to the same cohort's online questionnaire-based evaluations of their final-semester courses in 2020 (mean 18.8\%), which was much reduced compared to previous years' response rates (2016-2019 mean 55.9\%). This might indicate students' fatigue with the online learning environment.
A shortcoming of the approach as applied to research interviews was that it appeared to encourage minimal participation and very brief participant responses. Furthermore, the structured and asynchronous interview did not allow for any deeper exploration of the topic, as there was no exchange or conversation between researcher and participant. As only the pre-recorded interview questions were provided, this approach may not offer any additional benefits over an anonymous survey, and arguably, it might actively discourage research participation, as students could be identified in their video, prior to transcription and de-identification, which may have acted as a deterrent. This might in part account for the relatively low response rate in this research study. As such, the comments made by student participants may not be generalisable. While there appeared to be value in use of this platform in learning and teaching, it may not offer many advantages for use in research interviews.

\section{Limitations}

While the study aimed to interview a larger sample of students, recruitment was hampered by suspected student fatigue with online engagement and a potential lack of acceptance of the interface. In hindsight, synchronous face-to-face interviews using meeting or telecommunication platforms may have generated improved research participation. A clear limitation of this study was therefore the small sample size.

While the respondents were few, some important perceptions were identified regarding the novel use of asynchronous video interview software in lieu of face-toface OSCEs for both formative and summative learning. The positive aspects of the approach identified provide strong support for the use of this as a possible adjunct to traditional learning methods and warrant further research investigation.

\section{Future considerations}

This research highlighted the potential application of asynchronous video interviews platforms for use in OSCEs, particularly in formative learning. Future studies could investigate the approach in more junior students, to explore whether the platform could enhance their confidence and self-reflective skills in communication. Additionally, research could explore whether scaffolding skill development via asynchronous OSCEs prepares 
students for improved outcomes in higher fidelity synchronous OSCEs in later years.

The Big Interview platform enables use of Artificial Intelligence (AI) for first pass review of interviews and could potentially be employed to mark or provide feedback to student participants on their communication skills. The Al review evaluates video recordings for the participant's pace of speech, vocabulary, tone, volume, authenticity and counts pauses and disfluencies, such as 'ums' or filler words (Skillful Communications Inc., 2020). However, the summative marking in this study was completed traditionally, through the watching of all videos by a pharmacist academic with marking against a preprepared rubric. The Al capabilities of Big Interview or other asynchronous video interview software platforms could be explored in future research, to consider their suitability for academic use in pharmacy or other health professional OSCES.

Further research needs to be undertaken to determine the value and utility of asynchronous video interview platforms versus traditional qualitative research data collection methods.

\section{Conclusion}

Participating in virtual OSCEs using a novel approach in asynchronous video interview software may provide a valuable alternative to virtual OSCE teaching, allowing students to self-reflect and self-correct as they independently practise their patient questioning and counselling communication skills. While engagement was low, for those who did participate the interface was reported to be easy and comfortable to use. It may serve as a useful and sustainable adjunct to other learning and teaching methods in clinical communication. As a research tool, asynchronous video interview platforms are yet to be established as a useful data collection method.

\section{References}

Ali, M. (2020). What now and what next? The new era of OSCE: Innovation in learning assessment. Pharmacy Education, 56-58. https://doi.org/10.46542/pe.2020.202.5658

Archibald, M.M., Ambagtsheer, R.C., Casey, M.G., \& Lawless, M. (2019). Using Zoom videoconferencing for qualitative data collection: perceptions and experiences of researchers and participants. International Journal of Qualitative Methods, 18. https://doi.org/10.1177/1609406919874596
Austin, Z., O'Byrne, C., Pugsley, J., \& Munoz, L.Q. (2003). Development and validation processes for an objective structured clinical examination (OSCE) for entry-to-practice certification in pharmacy: the Canadian experience. American Journal of Pharmaceutical Education, 67(3), 76.

Australian Pharmacy Council Ltd. (2020). Accreditation Standards for Pharmacy Programs in Australia and New Zealand (online). Available from: https://www.pharmacycouncil.org.au/resources/ pharmacy-program-standards/

Bowers, R., Tunney, R., Kelly, K., Mills, B., Trotta, K., Wheeless, C. N., \& Drew, R. (2017). Impact of standardized simulated patients on first-year pharmacy students' knowledge retention of insulin injection technique and counseling skills. American Journal of Pharmaceutical Education, 81(6), 113. https://doi.org/10.5688/ ajpe816113

Education Management Solutions. (2020). Education Management Solutions (EMS) (online). Available from: https:// www.simulationiq.com/

Gorman, C.A., Robinson, J., \& Gamble, J.S. (2018). An investigation into the validity of asynchronous web-based video employment-interview ratings. Consulting Psychology Journal: Practice and Research, 70(2), 129. https://doi.org/10.1037/ cpb0000102

Higbea, A., Bald, E., Isaacs, A.N., Richter, S.K., Stamm, P.L., \& Kassel, L.E. (2020). Forging Ahead from Adaptations of Teaching during the COVID-19 Pandemic: Perspectives from Multiple Pharmacy Programs. Journal of the American College of Clinical Pharmacy. https://doi.org/10.1002/jac5.1349

Hsieh, H.-F., \& Shannon, S.E. (2005). Three approaches to qualitative content analysis. Qualitative health research, 15(9), 1277-1288. https://doi.org/10.1177/1049732305276687

Kakadia, R., Chen, E., \& Ohyama, H. (2020). Implementing an online OSCE during the COVID-19 pandemic. Journal of dental education. https://doi.org/10.1002/jdd.12323

Lara, S., Foster, C.W., Hawks, M., \& Montgomery, M. (2020). Remote Assessment of Clinical Skills during COVID-19: A Virtual, High-Stakes, Summative Pediatric OSCE. Academic Pediatrics. https://doi.org/10.1016/j.acap.2020.05.029

Major, S., Sawan, L., Vognsen, J., \& Jabre, M. (2020). COVID-19 pandemic prompts the development of a Web-OSCE using Zoom teleconferencing to resume medical students' clinical skills training at Weill Cornell Medicine-Qatar. BMJ Simulation and Technology Enhanced Learning. https://doi.org/10.1136/ bmjstel-2020-000629

Maloney, S., Storr, M., Morgan, P., \& llic, D. (2013). The effect of student self-video of performance on clinical skill competency: a randomised controlled trial. Advances in Health Sciences Education, 18(1), 81-89. https://doi.org/10.1007/ s10459-012-9356-1 
Marshall, C. (2017). Montessori education: a review of the evidence base. npj Science of Learning, 2(1), 1-9. https://doi.org/ 10.1038/s41539-017-0012-7

Motycka, C. A., St Onge, E. L., \& Williams, J. (2013). Asynchronous versus Synchronous Learning in Pharmacy Education. Journal of Curriculum and Teaching, 2(1), 63-67. https://doi.org/10.5430/ jct.v2n1p63

Mylrea, M.F., Sen Gupta, T., \& Glass, B.D. (2017). Developing professional identity in undergraduate pharmacy students: a role for self-determination theory. Pharmacy, 5(2), 16. https:// doi.org/10.3390/pharmacy5020016

Rahman, N., Nazar, N., \& Elnaem, M. (2020). Experiential learning in community pharmacy: Online and remote teaching experience in Malaysian higher education: Innovation in experiential learning or assessment. Pharmacy Education, 29-30. https:// doi.org/10.46542/pe.2020.202.2930

Rubinstein, P. (2020). Asynchronous video interviews: The tools you need to succeed (online). Available from: https:// www.bbc.com/worklife/article/20201102-asynchronous-videointerviews-the-tools-you-need-to-succeed

Skillful Communications Inc. (2020). Big Interview (online). Available from: https://biginterview.com/

Ytterberg, S. R., Harris, I. B., Allen, S. S., Anderson, D. C., Kofron, P. M., Kvasnicka, J. H., McCord, J.P., \& Moller, J.H. (1998). COGNITION, CONFIDENCE, AND CLINICAL SKILLS: Clinical Confidence and Skills of Medical StudentsUse of an OSCE to Enhance Confidence in Clinical Skills. Academic Medicine, 73(10), S103-105. https://doi.org/10.1097/00001888-199810000-00060 